\title{
ANALOG MITIGATION OF OUT OF BAND STRONG INTERFERERS IN WIDE BAND ACQUISITION FOR MULTIBAND HF TRANSMISSIONS
}

\author{
S. Zazo*, J.L. Sanz-González*, B. Pérez-Díaz ${ }^{\dagger}$, I. Pérez-Álvarez ${ }^{\dagger}$, J. López-Pérez ${ }^{\dagger}$, \\ L. Melián-Gutiérrez ${ }^{\dagger}$

\begin{abstract}
*Universidad Politécnica de Madrid (UPM), Dpto. SSR, E.T.S. Ingenieros de Telecomunicación, Ciudad Universitaria, 28040
Madrid, Spain, e-mail: santiago@gaps.ssr.upm.es

$\dagger$ Instituto para el Desarrollo Tecnológico y la Innovación en Comunicaciones (IDeTIC), Universidad de las Palmas de G. C.
\end{abstract} \\ Campus de Tafira, 35017 Las Palmas G.C. Spain, e-mail: iperez@idetic.eu
}

\begin{abstract}
Keywords: HF Modem, Wideband Transmission, Interference Mitigation, Zero-Crossing Statistics, Variable Notch Filter.
\end{abstract}

\begin{abstract}
It is clear that in the near future much broader transmissions in the HF band will replace part of the current narrow band links. Our personal view is that a real wide band signal is infeasible in this environment because the usage is typically very intensive and may suffer interferences from all over the world. Therefore, we envision that dynamic multiband transmissions may provide better satisfactory performance. From the very beginning, we observed that real links with our broadband transceiver suffered interferences out of our multiband but within the acquisition bandwidth that degrade the expected performance. Therefore, we concluded that a mitigation structure is required that operates on severely saturated signals as the interference may be of much higher power. In this paper we address a procedure based on Higher Order Crossings (HOC) statistics that are able to extract most of the signal structure in the case where the amplitude is severely distorted and allows the estimation of the interference carrier frequency to command a variable notch filter that mitigates its effect in the analog domain.
\end{abstract}

\section{Introduction}

Our group has been working on HF transmissions for several years developing a proprietary system denoted as HFDVL (HF Data + Voice Links) that permits interactive digital voice transmission along with data communications reaching data rates up to $8640 \mathrm{bps}$ in the $3 \mathrm{kHz}$ bandwidth, as described in several publications (see for instance [7]). The performance of the modem has been improving over the last years, especially with the addition of multiple antennas at receiver, known as Single Input Multiple Output (SIMO) system [5, 3]. At this point we are dealing with a promising feature that uses much broader bandwidths than current systems. We envisioned this challenging task several years ago and in fact we built our own broadband transceiver (up to $1 \mathrm{MHz}$ ) [4]. However, we do not think that standard broadband communication systems might be feasible in this scenario because with high probability there will be many interferences that will degrade the expected performance unless a broadband licensed band is used. We have noticed that some important steps have been taken in this direction, for instance, [1] describes some of the features of this tendency in the framework of the MIL-STD-188-141C, which is likely to include specifications for HF radio passbands of (nominally) 6, 12, and $24 \mathrm{kHz}$ with data rates up to $64 \mathrm{kbps}$. Also, there are already commercial transceivers including the 4-ISB mode as a transition phase to this concept. With a different perspective, the authors of this paper have developed some years ago our own transceiver capable of transmitting simultaneously a set of up to 16 narrow band signals within 1 $\mathrm{MHz}$ bandwidth [4]. Therefore, we envision that dynamic multiband transmissions may provide a very satisfactory performance where the number and positions of the multiple subbands will depend on the desired data rate or Quality of Service along with the instantaneous status of the channel in terms of propagation conditions and presence of interferences. We have learned from our experience with real broadband links that the presence of strong transmissions out of our set of channels, but still within the receiver wide band acquisition range, degrades significantly the expected performance for the current propagation characteristics. This effect comes from the reduction on the effective number of bits used to represent the desired signal because the Automatic Gain Control (AGC) intends to accommodate the aggregate received power in the ADC (Analog to Digital Converter) dynamic range. The consequent increase of the quantization noise dominates the thermal noise component and makes the system occasionally infeasible.

As this effect is generated before the ADC itself, we can not resort to any digital process to mitigate it and thus it has to be done in the analog domain. In this paper we will consider that we have single antenna reception and therefore we have just the frequency domain to cope with it (an accompanying paper will address this issue from beamforming principles [8]). The basic idea here is to design an estimator of the strongest interferer in order to tune an analog filter that eliminates it before the sampling process. 
The Mitigation Phase Block is the core of this proposal with the final objective of providing the fastest estimation of the interference channel to properly tune the Variable Notch Filter, and let the standard Receiver operate under desirable conditions. However, some particular issues have to be taken into account: on one hand, aggregated signals when a strong interference is present saturates completely the AGC and therefore the power spectrum density is spread. In this situation, standard spectrum estimates may fail. From our perspective, we have evaluated the use of HOC statistics [2] in order to relate the number of zero-crossings with the instantaneous frequency of the signals (observe that this signal feature is not affected by the saturation process). We have observed that this approach works properly not only for pure sinusoids but also for modulated signals. On the other hand, prompt detection is required in order to mitigate the degradation in much shorter time than the interleaving length selected in the modem and therefore enables the coding structure to correct burst errors.

The paper is structured as follows: in section 2 we will present our proposal for the Mitigation procedure while in section 3 we address some simulations to illustrate some results. Finally, section 4 sets some conclusions and forthcoming work.

\section{Mitigation procedure in the frequency domain}

The general approach is described in the Figure 1 showing schematically the complex base band spectra of involved signals in the range $[0, B] \mathrm{Hz}$ where for simplicity one desired signal and just one interferer are sketched:

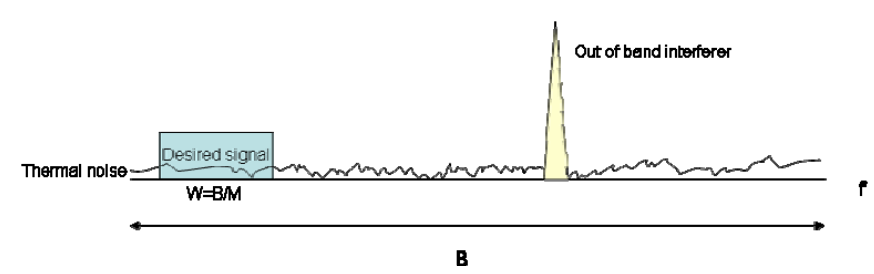

Figure 1. Simplified schematic view in broadband acquisition.

It is important to remark that we prefer to define this process as mitigation instead of cancellation because the final goal is to reduce the amount of interference and let the ADC work under proper conditions. If the signals are digitalized with the expected number of bits, all interferences will be eliminated by digital filtering in a later stage. Therefore, the first issue to take into account in this process is that it has to be designed to operate before the signal digitalization. In this paper, we will just explore the mitigation in the frequency domain controlling a variable tuning notch filter (as already mentioned, see [8] if interested in alternative procedures in the spatial domain).

Figure 2 shows the approach that we want to describe: in the lower part of the figure we have the standard receiver. When the switch is in position $A$, we can observe the performance of the standard approach. When the switch is in position $B$, the receiver processes a filtered signal with the interference already mitigated.

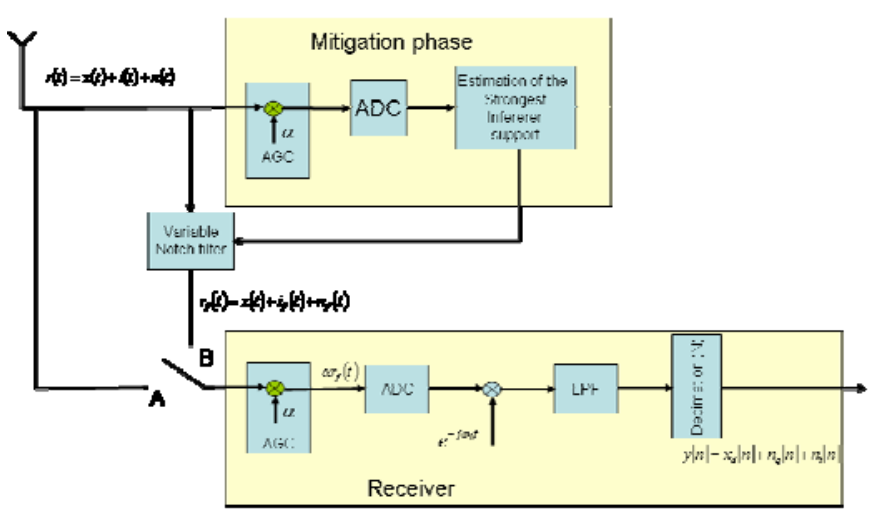

Figure 2. Receiver with the mitigation phase

Main involved parameters are the following (this analysis is included in full detail in an accompanying paper [6]):

$B$ : acquisition bandwidth. We suppose that the original bandpass signal is shifted to generate a complex base band version.

$W=B / M$ : desired signal bandwidth. We suppose an integer ratio $(M)$ between the bandwidth acquisition and $W$ to simplify the decimation process. Indeed it is realistic in HF where typically $\mathrm{W}=3 \mathrm{kHz}$ and $M$ will represent the number of standard channels within the captured bandwidth.

$x(t)$ is the desired bandlimited signal characterized by a certain power $\left(\sigma_{x}^{2}\right)$ and a certain Peak to Average Ratio (PAR) denoted as $P A R_{x}$ and defined as:

$$
P A R_{x}=\frac{\max \left(|x(t)|^{2}\right)}{\sigma_{x}^{2}}
$$

$n(t)$ is the thermal noise characterized as an additive white (within the acquisition band) Gaussian noise with flat spectrum density $N_{0}$. The power becomes $\sigma_{n}^{2}=B N_{0}$.

$i(t)$ is the interference bandlimited signal characterized by a certain power $\left(\sigma_{i}^{2}\right)$ and a certain $P A R_{i}$.

$r(t)=x(t)+i(t)+n(t)$ is the aggregated signal.

Let us start remark with an example what is the main challenge that we face in this scenario. Figure 3 shows the Power Spectrum Density (PSD) where a BPSK signal transmitted in the $9-12 \mathrm{kHz}$ band is received with the interference of a much stronger BPSK in the $72-75 \mathrm{kHz}$ channel. This PSD before the ADC matches what is expected where clearly both spectra are distinguishable. Any spectrum estimation procedure, where probably the periodogram using the FFT algorithm is the simplest one, will provide accurate information about the location of the interference. In this favourable case, a standard notch filter will eliminate the interference.

In standard operating mode, the AGC of the receiver is matched to the desired signal so that to expand its dynamic 
range to the maximum number of bits of the $\mathrm{ADC}$ and therefore minimize the quantization effects. If the interference appears, it typically may have much larger power (if comparable, the degradation is negligible as shown in [6]) because the interference transmitter could be quite close to the desired receiver. As the AGC is designed to have some inertia and track channel response variations rate (few Hertz in HF), it is not capable to response immediately to this new situation and thus the signal saturates (let us remark that even in the case where we have an ideal AGC to avoid saturations, this behaviour is not desirable because the number of bits dedicated to the desired signal will be few than needed, and performance will in any case degrade).

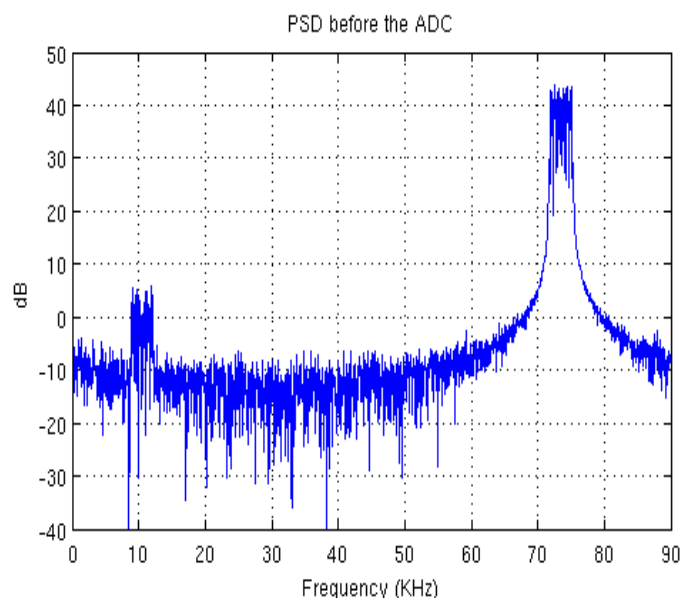

Figure 3. Received PSD before the ADC

The point that we want to emphasize now is the fact that this saturation clips the aggregated signal spreading out the original PSD. Figure 4 shows how the spectra in Figure 3 are distorted after the ADC saturation.

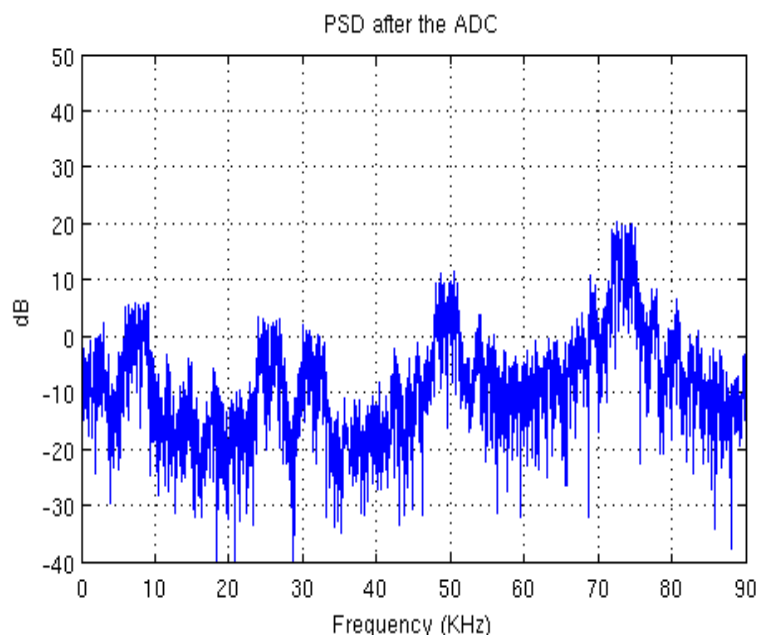

Figure 4. Received PSD after the ADC

Therefore, prompt frequency estimation is not an easy task. Standard approaches will require a fast AGC that reconstructs the original spectrum at the output of the ADC but it may take unaffordable time for convergence and estimation.

As an alternative, we have evaluated the use of HOCs statistics [2] in order to relate the number of zero-crossings with the instantaneous frequency of the signal (observe that this signal feature is not affected by the saturation process) and found it working properly not only for pure sinusoids but also for modulated signals. Therefore, this statistical analysis is robust in these scenarios and will play an important role in this approach. Let us now describe in more detail the Mitigation Phase Block presented in Figure 2.

\subsection{Acquisition procedure}

The mitigation process will be accomplished in the digital domain in order to make simpler and efficient algorithms. Therefore, an extra ADC is needed. However, this device is not required to operate with similar number of bits as the $\mathrm{ADC}$ of the receiver. In this case, as the signal will be saturated, we just take care on the information related to the polarity to determine the zero-crossings. Therefore, as it is showed in Figure 5 this extra ADC just needs to provide a one bit signal to distinguish positive (1) and negative (0) voltages working at the same sampling frequency of the main ADC of the broadband receiver. This sequence of $\{0 \mathrm{~s}, 1 \mathrm{~s}\}$ is all that is needed for the frequency estimation process which will be detailed below.

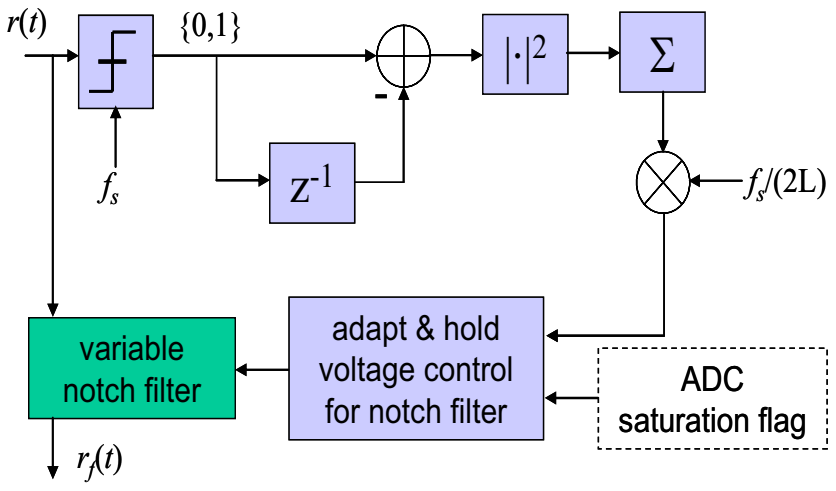

Figure 5. Block diagram of 1-bit frequency estimator

Once the frequency is estimated the voltage control for the notch filter is adapted and sourced to the variable notch filter if interference is present. Early alert of its presence will be given by the saturation flag of the main ADC. In this way all the process is simple and we guarantee a very short time for the interference mitigation.

\subsection{Estimation of interference in the HOC domain}

Once we have the sequence of $\{0 \mathrm{~s}, 1 \mathrm{~s}\}$, we need to estimate the instantaneous frequency of the interference. In [2] there is a variety of methods but in this paper we just describe a simple one with very low computational requirements.

$$
\hat{f}=\frac{\sum_{n=1}^{L}|x(n)-x(n-1)|^{2}}{L} \frac{f_{s}}{2}
$$


where the numerator is the number of zero-crossings, $f_{s}$ represents the sampling frequency and $x(n) \in\{0,1\}$ is the output of the acquisition process as described in section 2.1. It can be observed that (2) represents a batch implementation size $L$ but iterative procedures also work quite well converging very fast and thus allowing prompt estimation. This issue is very important because if we intend to have stable transmissions it is highly undesirable to break a link while the interference appears and restart the transmission in a different band. As standard interleaving length is about 5 seconds, the maximum convergence time should be a small fraction of it. Fortunately, we will show in the next section that this is the case indeed.

We may remark that the desired signal is negligible with respect the interference, thus it will not affect this estimation process.

\subsection{Tuning a variable notch filter}

Once the carrier frequency has been estimated, this information is provided to tune a variable notch filter. Although we are still far from desirable implementations, we have considered some alternatives using commercial devices. Taking into account our broadband digital HF transceiver design [4] (double-conversion super-heterodyne), the best place to put this filter is at the second IF output $(10.7 \mathrm{MHz})$, just where AGC loop takes its sample and just before main ADC. This variable notch filter must meet the following requirements: tunable range from $10.2 \mathrm{MHz}$ to $11.2 \mathrm{MHz}$, low insertion loss $(<3 \mathrm{~dB})$, narrow stopband bandwidth $(<$ $100 \mathrm{kHz}$ ) and more than $10 \mathrm{~dB}$ of interference rejection (notch depth). We consider that at least $10 \mathrm{~dB}$ of interference rejection ratio is necessary to avoid most of ADC artifacts.

There are several implementations of notch filters, although most of them are focused on low frequencies $(<1 \mathrm{MHz})$ and microwave bands (cavities). The most used topology is the Twin- $T$ notch filter, that is implemented using resistors and capacitors and operational amplifiers to boost the $Q$ factor (i.e. $3 \mathrm{~dB}$ bandwidth). Although it could obtain a high $Q$, the main limitation is the operational amplifier bandwidth, that must be more than 100 times the filter central frequency ( $1 \mathrm{GHz}$ in our case).

Our chosen implementation is using coils and capacitors with different filter notch topologies. We can tune the filter using a varactor diode in different points instead of capacitors. The main limitation in this kind of circuits is the components unloaded $Q$ that are normally very low and therefore results in broader filter notch bandwidth. We are working in some techniques to improve the $Q$ factor.

Currently, our filter is able to tune from $10.2 \mathrm{MHz}$ to $11.2 \mathrm{MHz}$ with $250 \mathrm{kHz}$ of $6 \mathrm{~dB}$ bandwidth. Although we continue working on it to improve filter characteristics, it can change frequencies in 10 miliseconds.

\section{Simulations}

The scope of this paper is limited to simulated signals and implementations leaving for future developments the evaluation with real hardware and real time software.
To evaluate the performance and limitations of these ideas we have built a simulator of the broadband acquisition problem. We have defined a certain number of $3 \mathrm{kHz}$ bandwidth channels $\left(N_{c}=30\right.$ in the simulations to be shown) sampled at $f_{s}=90 \mathrm{kHz}$ assuming base band complex acquisition. We can place the desired signal power normalized in any channel $\left(c_{d}=4\right.$ in these simulations as shown in Figure 3$)$ and the interference is located in different channels as a parameter $\left(c_{i}=25\right.$ as an example in Figure 3$)$ with parameterized power. We can select different signals ranging from pure sinusoids to single or multicarrier digital transmissions. In this paper we are going to report just on the behaviour where both signals are BPSK (with raised cosine pulse shape roll-off 0.3 ) for simplicity, but conclusions can be extended to other modulations. We assume that the AGC works properly so the ADC dynamic range accommodates the maximum signal amplitude variations with any distortion including the thermal noise. The ADC is represented by the number of bits with allequal steps. In this case, as an illustrative example, 10 bits has been assumed where 2 bits $(6 \mathrm{~dB})$ are reserved for headroom (signal and channel variations) and 8 for signal conversion (therefore, $N_{b}=8$ in simulations).

The first point that we have evaluated is the effect in terms of BER degradation due to the presence of the interference. We will consider just the AWGN although extensions to faded channels are straightforward. This example has already been shown in Figure 3 using the Welch procedure to estimate the PSD. The nonlinear effect of the clipping process at the ADC illustrated in Figure 4 remarks the high distortion introduced that affects the desired signal.

In terms of BER, we show in Figure 6 that for a $\mathrm{SNR}=10 \mathrm{~dB}$ (quite reasonable in HF communications) it is observed how the system degrades depending on two parameters. $x$-axe represents the interference power while different curves are parameterized according to the interference proximity in the channel allocation.

In can be noticed that the presence of the interferer very close to the desired signal $\left(c_{d}=4, c_{i}=5\right)$ in the blue line degrades the performance even for small interference power. For the case where $\left(c_{d}=4, c_{i}=10\right)$ in green line and $\left(c_{d}=4, c_{i}=15\right)$ in red line the performance starts from the theoretical value $(\mathrm{SNR}=10$ dB)

$$
B E R=Q(\sqrt{s n r})=7.82 \times 10^{-4}
$$

but immediately degrades when the interference becomes more important.

A second set of simulations represent the evolution of the frequency estimator. In Figure 7 we show the evolution of the case represented in Figures 3 and $4\left(c_{d}=4, c_{i}=25\right)$ in order to remark that in about 0.15 seconds the estimator has converged. Let us remark that in this situation, we are not really interested in accuracy but in convergence speed. Dashed lines represent the limits of the channelization $f_{0} \pm 1.5 \mathrm{kHz}$ that will determine in which channel the interferer is located. 


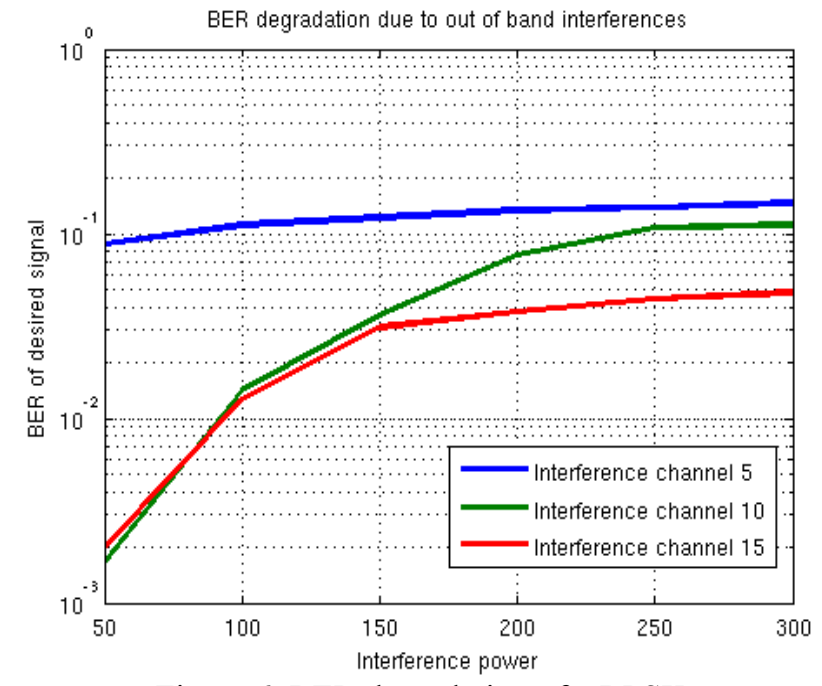

Figure 6. BER degradation of a BPSK

Very interestingly, 0.15 seconds is far bellow the interleaving length (even for short interleaving about 1 second long) therefore it is expected that the coder will cope with the error burst generated until the mitigation process has converged.

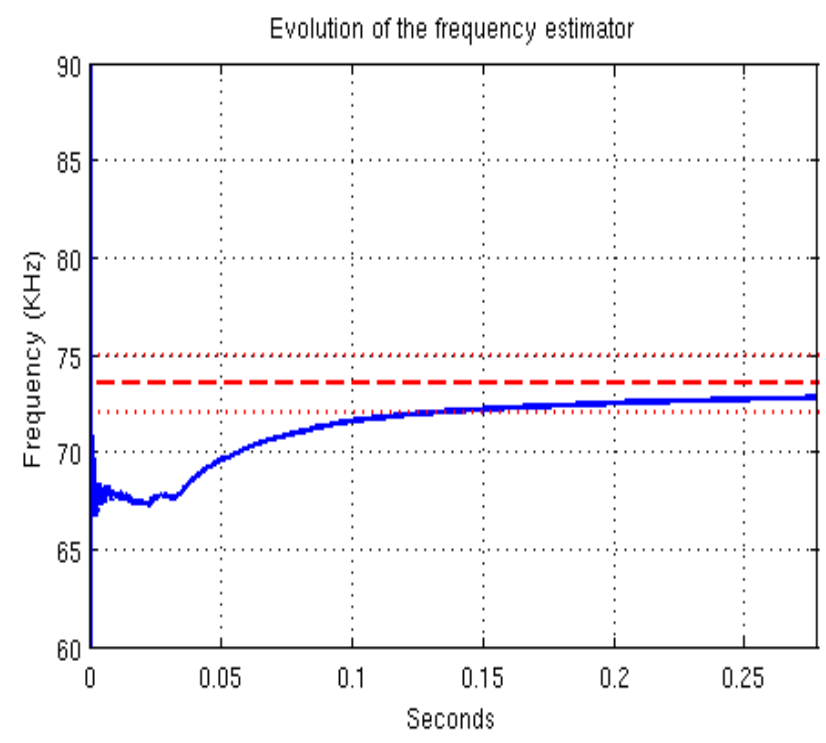

Figure 7. Evolution of the Frequency Estimator using HOCs

\section{Conclusions}

In this paper we have emphasized that broadband acquisitions might require an extra block specially designed to mitigate interferences within the bandwidth. We have remarked that this procedure must operate in the analog domain and must be able to estimate PSDs of strongly distorted signals suffering from saturation effects. Higher Order Crossing statistics have shown to be a powerful technique to cope with this problem with low cost implementation and high performance. Other alternatives described in [2] and related papers point out the importance of these techniques based on Order Crossings and the potential use of more robust / faster convergence of several alternatives not addressed in this paper.
Finally, we wish to emphasize that the scope of this paper has been to accomplish a preliminary study as a proof of concept using simulated signals and procedures. In the future we plan to build the hardware and real time software to show in real links these capabilities inside the HFDVL framework.

\section{Acknowledgements}

This work was supported by the Spanish Ministry of Science and Innovation under grants TEC2010-21217-C02 CR4HFDVL, and CONSOLIDER-INGENIO 2010 CSD2008-00010 COMONSENS.

\section{References}

[1] E. E. Johnson. Performance Envelope of Broadband HF Data Waveforms, (WBHF Applications), Proceedings of MILCOM 2009, IEEE*, Boston, MA, Oct 2009.

[2] B. Kedem. Time Series Análisis by Higher Order Crossings. IEEE Press, New York 1994.

[3] Peco, C.; Zazo, S.; Pérez-Álvarez, I.; López-Pérez, J. On the diversity in multiantenna HF communications, IRST 2009: 11th International Conference on Ionospheric Radio Systems \& Techniques. Edinburgh, U.K.; April 2009.

[4] B. Pérez-Díaz.; Jiménez-Yguacel, E.; López-Pérez, J.; Pérez-Álvarez, I.; Zazo-Bello, S.; Medieta-Otero, E. Design and construction of a broadband (1MHz) digital $\mathrm{HF}$ transceiver for multicarrier and multichannel modulations, IRST 2009: 11th International Conference on Ionospheric Radio Systems \& Techniques. Edinburgh, U.K.; April 2009.

[5] I. Pérez-Álvarez, S. Zazo, M. Ghogho, J. López-Pérez, Experimental Results on Multicarrier MIMO HF Communications". 2011 URSI General Assembly and Scientific Symposium (GASS), Istanbul, Turkey, August 13-20, 2011.

[6] B. Pérez-Díaz, S. Zazo, I. Pérez-Álvarez, J. López-Pérez, L. Melián-Gutiérrez, E. Jiménez-Yguacel. Theory and Practice for Modelling the Broadband Acquisition in HF Transmissions. IRST 2012: 12th International Conference on Ionospheric Radio Systems \& Techniques. York, U.K.; May 2012.

[7]: H. Santana; Zazo, S.; Pérez, I.; Raos, I.; Mendieta, E.; López, J.; Validation of a HF Spread Spectrum MultiCarrier Technology through real Link Measurements. European Transactions on Telecomunications. Vol.:17, pp.651-657, Nov.-Dec. 2006.

[8] S. Zazo, I. Pérez-Álvarez, J. López-Pérez, B. Pérez-Díaz, E. Jiménez-Yguacel, L. Melián-Gutiérrez, J.L. SanzGonzález. Spatial Domain Mitigation of Out of Band Strong Interferers in HF Wide Band Acquisition using Analog Beamforming Principles. IRST 2012: 12th International Conference on Ionospheric Radio Systems \& Techniques. York, U.K.; May 2012. 